\title{
O papel dos antipsicóticos atípicos no tratamento do transtorno bipolar: revisão da literatura
}

\author{
The role of atypical antipsychotic agents in the treatment of bipolar \\ disorder: a literature review
}

\author{
Acioly LT Lacerda a,b, Jair C Soares a e Mauricio Tohen ${ }^{c, d}$ \\ a Department of Psychiatry, Western Psychiatric Institute and Clinic, University of Pittsburgh School of Medicine. Pittsburgh, PA, EUA. ${ }^{\mathrm{b}}$ Departa- \\ mento de Psicologia Médica e Psiquiatria da Faculdade de Ciências Médicas da Unicamp. Campinas, SP, Brasil. 'Lilly Research Laboratories. \\ Indianápolis, IN, EUA. ${ }^{\mathrm{d} D e p a r t a m e n t}$ of Psychiatry, Harvard Medical School, McLean Hospital. Belmont, MA, EUA
}

Resumo Estudos recentes têm demonstrado que a eficácia do lítio é significativamente inferior à descrita pelos primeiros trabalhos, embora ainda seja a medicação de referência no tratamento do transtorno afetivo bipolar. Apesar de um perfil de segurança desfavorável, os antipsicóticos clássicos sempre apresentaram um papel importante no tratamento desse transtorno psiquiátrico, especialmente como coadjuvante em sua fase maníaca aguda. Os autores, utilizando informação obtida no Medline, fizeram uma revisão acerca do papel dos antipsicóticos atípicos no tratamento dos pacientes bipolares. Baseado nos dados da literatura, a olanzapina mostrou-se bastante eficaz no manejo da mania aguda, quando uma média de $63,5 \%$ dos pacientes apresentaram melhora significativa em estudos duplo-cego controlados, apresentando ganho de peso como único efeito colateral relevante. A clozapina e, mais ainda, a risperidona apresentaram dados menos consistentes, grande parte em função de deficiências metodológicas dos poucos estudos conduzidos até o presente estudo. Os dados preliminares relativos à eficácia desse grupo farmacológico nos quadros refratários e nos sintomas depressivos são promissores, mas ainda não definitivos. Em relação a seus efeitos potenciais como estabilizadores do humor, não existem evidências conclusivas oriundas de estudos controlados, mas há interesse considerável para realização de investigações em pacientes bipolares tratados com antipsicóticos atípicos por períodos de tempo mais prolongados. Pesquisas futuras poderão tornar mais claras essas possíveis características terapêuticas.

Descritores Transtorno bipolar. Planejamento de assistência ao paciente. Agentes antipsicóticos. Neurolépticos. Revisão.

Abstract Even though lithium is still the choice drug in the treatment of bipolar disorder, recent studies have shown that it has a significant lower efficacy than previously described in earlier studies. Despite its adverse side effects, typical antipsychotic agents have often had a prominent role in the treatment of this psychiatric disorder, especially in the acute manic phase. Recently new alternatives have become available with the development of newer atypical antipsychotic agents. A comprehensive Medline search was conducted, and all available literature concerning the role of atypical antipsychotics in the treatment of bipolar patients was retrieved. Olanzapine showed to be quite effective in the treatment of acute mania, and it was found that an average of $63.5 \%$ of the patients had a significant improvement in double blind controlled studies, with weight gain as the major side effect. Data was less robust for clozapine and risperidone, mainly due to methodological limitations of the few available studies. It was also found a considerable interest in future investigating the efficacy of these pharmacological agents in refractory cases and in the treatment of the disorder's depressive phase. Additionally, there has been extensive interest in evaluating their potential action as mood stabilizers, for which there will be a need of longer-term longitudinal studies.

Keywords Bipolar disorder. Patient care planning. Antipsychotic agents. Neuroleptics. Review. 


\section{Introdução}

O lítio é a mais antiga medicação aprovada e a mais amplamente estudada para o tratamento do transtorno bipolar, seja na fase aguda ou na de manutenção. Porém, ao contrário das primeiras impressões, não há resposta para essa medicação em um número substancial de pacientes, e também há pacientes que não toleram seus efeitos adversos. ${ }^{1,2}$ Peselow et al, ${ }^{3} \mathrm{em}$ um estudo de seguimento, observaram que, durante a fase de manutenção com o lítio, $17 \%$ dos pacientes bipolares tiveram uma recaída no primeiro ano, $48 \%$, após três anos, e $63 \%$, após cinco anos. Outros autores também relatam taxas de $50 \%$ a $60 \%$ de pacientes bipolares com resposta inadequada à monoterapia com o lítio. ${ }^{1,4,5}$

Por outro lado, anticonvulsivantes como valproato e carbamazepina parecem apresentar maior eficácia do que o lítio em alguns subgrupos específicos, como estados mistos, pacientes com achados neurológicos associados, história familiar negativa, presença de disforia e de ansiedade intensas e ciclagem rápida. ${ }^{6,7}$ Alguns autores também descrevem tais medicações como comparáveis ao lítio em quadros típicos de mania aguda. ${ }^{8-10}$

Com o mesmo intuito de buscar novas alternativas de tratamento para os pacientes que não se beneficiam ou não toleram o lítio, alguns autores avaliaram a eficácia de benzodiazepínicos, como clonazepam e lorazepam, no tratamento de pacientes bipolares. Embora com achados pouco consistentes, os estudos disponíveis sugerem alguma utilidade, como medicações coadjuvantes no tratamento da mania aguda, porém ressaltam riscos de seu uso (indução ou agravamento de agitação psicomotora em alguns pacientes, risco de abuso do uso dessas substâncias ou recaída quando há abuso no uso de outras substâncias, comorbidade comum no transtorno bipolar). ${ }^{11-16}$

Outro fator relevante que tem sido pouco avaliado em estudos sistematizados é o tempo médio para o início de ação das diversas medicações. Diversos estudos sugerem que o lítio é comparável e, possivelmente, superior aos antipsicóticos no tratamento em curto prazo da mania aguda (exemplo: três a seis semanas de tratamento). Sugerem também que os antipsicóticos podem ter início de ação mais rápida na mania; logo, podem ser mais efetivos inicialmente (exemplo: na primeira semana de tratamento), especialmente em pacientes gravemente doentes, agitados ou com sintomas psicóticos proeminentes. ${ }^{17,18}$

Desse modo, a busca por novas estratégias farmacológicas para o tratamento de pacientes bipolares é bastante justificável, seja pela grande parcela de pacientes que não responde adequadamente à medicação de referência seja pela busca de um perfil de efeitos colaterais e de segurança mais favorável seja pela busca de um início de ação mais rápido, o que é fundamental no tratamento de qualquer transtorno psiquiátrico, especialmente na mania aguda, ou seja, pela freqüente necessidade de associar uma medicação que potencialize uma melhora parcial com a monoterapia.

O uso de antipsicóticos clássicos no tratamento do transtorno bipolar se encontrava entre as primeiras e as poucas estratégias para a abordagem da mania aguda, assim como para a profilaxia. ${ }^{19}$ Surpreendentemente, apesar de seu perfil de efeitos colaterais ser bastante desfavorável, esse grupo de medica- ções continua sendo utilizado comumente nessas situações clínicas. ${ }^{20}$ Com o advento dos chamados antipsicóticos atípicos, que, dentre outras características, apresentam um risco bastante reduzido de induzir efeitos adversos de ordem neurológica, ${ }^{21}$ certamente se abre um largo horizonte, já que estes têm se mostrado tão eficazes quanto os antipsicóticos clássicos no tratamento dos sintomas positivos da esquizofrenia, além de mostrarem-se superiores quanto à cognição e aos sintomas negativos que, de certo modo, assemelham-se a alguns sintomas depressivos. ${ }^{22}$ Dessa forma, em teoria, os antipsicóticos atípicos poderiam ter efeitos terapêuticos na mania e na depressão, como sugerido em alguns estudos preliminares, ${ }^{23}$ fazendo com que esse grupo de medicações seja particularmente interessante no manejo farmacológico do transtorno bipolar.

O presente trabalho se propõe a fazer uma revisão crítica dos estudos publicados acerca do uso dos antipsicóticos atípicos no tratamento do transtorno bipolar. Por conta do maior número de estudos publicados e por ser o único antipsicótico atípico aprovado para o tratamento do transtorno bipolar, a olanzapina será enfatizada.

\section{O uso de antipsicóticos no tratamento do transtorno bipolar}

\section{Antipsicóticos clássicos}

Antes da generalização do uso do lítio, os antipsicóticos se encontravam entre as raras medicações disponíveis para o tratamento da mania aguda e para o tratamento de manutenção dos pacientes bipolares. ${ }^{19}$ Embora o uso de antipsicóticos no transtorno bipolar seja quase universal na prática clínica, especialmente no tratamento da mania aguda, poucos têm sido os estudos dirigidos à avaliação de sua eficácia. Pelo contrário, boa parte dos estudos teve como principal objetivo a avaliação de possíveis efeitos colaterais, apontando, portanto, para uma relação custo-benefício desfavorável. ${ }^{24}$

De fato, na prática clínica diária, o uso de antipsicóticos não tem se limitado ao tratamento da fase maníaca aguda, prolongando-se em muitos casos por vários meses após a remissão desta e, até mesmo, indefinidamente. Estudando um grupo de 77 pacientes bipolares, Keck et $\mathrm{al}^{20}$ relataram que cerca de $68 \%$ desses pacientes continuavam utilizando antipsicóticos após seis meses de seguimento. $\mathrm{O}$ uso de antipsicóticos nesse grupo, após seis meses de seguimento, correlacionou-se significativamente $(\mathrm{p}<0,05)$ à não-adesão ao tratamento no mês anterior à internação, ao sexo masculino, à sintomatologia maníaca mais grave quando da alta hospitalar e à prescrição dessas medicações à época da alta hospitalar. Serniak et $\mathrm{al}^{25}$ avaliaram, retrospectivamente, 49 pacientes bipolares saídos de internação psiquiátrica por um período de seis meses. Apenas $11 \%$ desses pacientes não receberam antipsicóticos durante o período de internação, ao passo que, após seis meses, apenas $5 \%$ dos pacientes que usaram antipsicóticos, quando da alta hospitalar, não mais faziam uso dessas medicações. Nessa época, embora com uma significativa redução da dose média de antipsicóticos (cerca de $20 \%$ ), o grupo continuava recebendo consideráveis doses de antipsicóticos (o equivalente a $634 \mathrm{mg} /$ dia de clorpromazina). 
Em estudo de metanálise, Tohen et al (2001) revisaram, a partir de pesquisa no Medline, 16 publicações referentes a um total de 2.378 pacientes bipolares. Como um todo, $84,7 \%$ dos pacientes receberam antipsicóticos clássicos $(90,7 \%$ dos pacientes internados e $63,3 \%$ dos pacientes ambulatoriais). Em cerca de $54 \%$ dos casos, os antipsicóticos típicos foram utilizados como monoterapia. A partir da informação acerca do tempo de exposição a antipsicóticos, em quatro estudos (perfazendo um percentual de $32 \%$ de todos os pacientes incluídos na metanálise), os autores concluem que o tempo mínimo médio de exposição foi de 2,5 meses.

Entretanto, tratando-se dos antipsicóticos clássicos, os riscos de efeitos colaterais graves, sejam agudos ou crônicos, estão longe de ser desprezíveis. Alguns autores relatam, inclusive, que pacientes bipolares apresentam um risco mais elevado para desenvolver discinesia tardia, ${ }^{26,27}$ certamente um dos efeitos colaterais mais grave e debilitantes desse grupo de medicações. Prevalências de discinesia tardia em pacientes bipolares expostos a antipsicóticos clássicos têm sido da ordem de $23 \%$ a $41 \%$, segundo alguns autores. ${ }^{26,28}$ Também, diversos estudos reconhecem a eficácia dos antipsicóticos clássicos no tratamento dos sintomas maníacos, porém sugerem que o tratamento de manutenção com essas medicações pode aumentar a freqüência de episódios depressivos. ${ }^{29,30}$

Soares et al, ${ }^{24}$ em recente trabalho de revisão acerca do papel dos antipsicóticos no tratamento do transtorno bipolar, concluem que, a despeito do importante progresso na psicofarmacologia do transtorno bipolar, os antipsicóticos ainda ocupam um lugar de importância, especialmente na condição de medicações coadjuvantes. Pelo menos para um subgrupo de pacientes refratários, os antipsicóticos parecem ser coadjuvantes necessários para a otimização do tratamento. Esses autores sugeriram que, como regra, caso o uso de antipsicóticos (clássicos) seja indispensável, eles devem ser utilizados pelo menor tempo e com as menores doses possíveis e que, para os pacientes que realmente necessitam destes por um longo período, devem ser monitorados cuidadosamente acerca de sinais precoces de discinesia tardia. No entanto, os autores ponderam que os estudos disponíveis acerca do uso de antipsicóticos tradicionais no transtorno bipolar são carentes de padronizações e informações controladas, especialmente quando são utilizados na fase de manutenção sem permitir conclusões mais definitivas.

\section{Antipsicóticos atípicos}

A clozapina foi primeiramente descrita como antipsicótico atípico por Hippius \& Angst, que se basearam unicamente em suas observações de que havia uma marcante diferença entre essa medicação e os antipsicóticos tradicionais no que se refere à incidência de efeitos colaterais como parkinsonismo, acatisia e distonia aguda. ${ }^{31}$ À mesma época, Meltzer et al propuseram uma classificação dos antipsicóticos, por meio da análise discriminatória de suas afinidades pelos receptores D1, D2 e 5-HT2A, de modo que os antipsicóticos atípicos apresentariam uma baixa afinidade pelo receptor D2 e uma elevada afinidade pelo receptor 5-HT2A, não havendo contribuição do receptor D1 nessa diferenciação. ${ }^{32}$
Segundo Remington \& Kapur, ${ }^{21}$ embora não haja um consenso acerca da conceituação de atipia, alguns critérios se encontram comumente presentes nos estudos desenvolvidos por diversos autores: baixa incidência de efeitos colaterais, como sintomas extrapiramidais e hiperprolactinemia, ao lado de uma maior eficácia no tratamento dos sintomas negativos da esquizofrenia. No entanto, é importante ressaltar que esse grupo de medicações, que inclui clozapina, risperidona, olanzapina, quetiapina, sertindole e ziprasidone, é bastante heterogêneo, mesmo havendo uma concordância em torno desses critérios de atipia.

Poucos foram os estudos controlados utilizando os antipsicóticos atípicos no tratamento dos transtornos afetivos. A olanzapina é o antipsicótico atípico mais bem estudado no tratamento do transtorno bipolar, tendo sido, inclusive, aprovada recentemente pelo Food and Drug Administration (FDA), assim como pelo Ministério da Saúde, no Brasil, para o tratamento da mania aguda.

\section{Clozapina}

Já em 1977, Muller \& Heipertz, ${ }^{33}$ em estudo aberto, observaram uma significativa melhora em um grupo de 52 pacientes com quadros maníacos agudos tratados com clozapina (metade deles, na forma de monoterapia). Os autores sugerem uma atividade antimaníaca intrínseca da clozapina ao lado de um rápido início de ação.

Em 1991, Calabrese et $\mathrm{al}^{34}$ relataram dois casos de cicladoras rápidas refratárias a tratamentos convencionais que responderam satisfatoriamente ao uso de clozapina. Pela avaliação de um período médio de administração de três meses, os autores sugerem que a clozapina apresentou ação antimaníaca e antidepressiva agudas e profiláticas. Seguiram-se relatos que confirmaram a impressão inicial desses autores. ${ }^{35-38}$

Em estudo aberto, Calabrese et al $^{39}$ avaliaram a eficácia da clozapina em dez pacientes bipolares e 15 com transtorno esquizoafetivo, todos resistentes ou intolerantes ao uso do lítio, da carbamazepina, do valproato e, pelo menos, dois antipsicóticos clássicos. No grupo como um todo, observou-se melhora de $50 \%$ ou mais na subescala de sintomas positivos da BPRS em 19 (76\%) dos 25 sujeitos. Apresentaram uma redução de mais de 50\%, no Young Mania Rating Scale (YMRS), 18 pacientes ( $72 \%$ da amostra).

Banov et al, ${ }^{40}$ em um estudo naturalístico, examinaram 52 pacientes bipolares, 81 com transtorno esquizoafetivo, 14 com depressão unipolar, 40 com esquizofrenia e seis com outros diagnósticos. Os sujeitos foram seguidos por um período médio de 18,7 meses após início da clozapina. Os autores registraram uma melhor evolução, pela avaliação do Clinical Global Impression (CGI), do Global Assessment Scale (GAS) e da taxa de reinternação, nos pacientes com quadros maníacos e esquizoafetivos do tipo maníaco, quando comparada a pacientes com quadros de depressão unipolar, bipolar ou esquizoafetivos do tipo depressivo.

Zarate et $\mathrm{al}^{41}$ avaliaram 11 pacientes com diagnóstico inicial de transtorno bipolar, fase maníaca, e seis pacientes com transtorno esquizoafetivo do tipo maníaco, usuários de clozapina 
como monoterapia, por um período médio de 16 meses. Durante o seguimento, considerou-se que 15 pacientes $(88 \%)$ responderam ao tratamento. Os autores concluem que a clozapina se mostrou útil como um tratamento de manutenção.

Em estudo aberto prospectivo, Calabrese et $\mathrm{al}^{42}$ avaliaram o uso da clozapina em 25 pacientes com mania aguda (dez bipolares e 15 esquizoafetivos do tipo bipolar) durante 13 semanas. Apresentaram uma acentuada redução nos escores da YMRS 18 (72\%) pacientes, enquanto oito (32\%) mostraram uma acentuada melhora na avaliação pela escala BPRS, com os pacientes bipolares apresentando uma redução significativamente maior nesses escores.

Ciaparelli et al, ${ }^{43}$ por um período de 24 meses, acompanharam 31 pacientes com esquizofrenia, 26 com transtorno esquizoafetivo e $34 \mathrm{com}$ transtorno bipolar e com sintomas psicóticos. Dentre os pacientes bipolares, 12 (35\%) utilizaram neurolépticos; oito $(23,5 \%)$, lítio; nove $(26,5 \%)$, anticonvulsivantes; 12 (35,3\%), antidepressivos; e um $(2,9 \%)$, benzodiazepínicos, como medicações concomitantes ao uso da clozapina. Os três grupos apresentaram uma significativa melhora, a qual se mostrou substancialmente maior no grupo de pacientes bipolares ( $83 \%$ responderam satisfatoriamente).

Em estudo naturalístico retrospectivo, Guille et $\mathrm{al}^{44}$ selecionaram 50 pacientes com transtorno bipolar I que foram concomitantemente tratados com clozapina, risperidona ou olanzapina. A eficácia, assim como a incidência de efeitos extrapiramidais, mostrou-se semelhante entre as três medicações.

Sintetizando, os estudos sugerem que a clozapina constitui uma estratégia útil no tratamento da mania aguda, especialmente nos quadros atípicos e refratários, embora tais achados se refiram a estudos não-controlados e, em boa parte, retrospectivos, o que limita em muito a formulação de qualquer conclusão.

\section{Risperidona}

Keck et $\mathrm{al}^{45}$ avaliaram retrospectivamente a eficácia da risperidona no tratamento de pacientes com diagnóstico de esquizofrenia $(n=49)$, transtorno esquizoafetivo do tipo bipolar $(n=58)$ e do tipo depressivo $(n=23)$, transtorno bipolar $(n=11)$ e depressão com sintomas psicóticos $(n=3)$. Todos os pacientes bipolares apresentaram melhora moderada ou acentuada, embora todos estivessem usando, concomitantemente, estabilizadores do humor.

Estudando dez pacientes bipolares, Schaffer \& Schaffer ${ }^{46}$ observaram uma resposta satisfatória ao tratamento com risperidona em cinco deles (50\%): três apresentaram uma exacerbação do quadro maníaco/hipomaníaco, achado relatado em outros trabalhos, ${ }^{47,48}$ e dois não toleraram os efeitos colaterais. Os autores relatam que vários pacientes usavam concomitantemente estabilizadores do humor.

McIntyre et $\mathrm{al}^{49}$ relataram uma melhora leve a moderada em quatro de sete pacientes bipolares, em cujo regime de medicação (estabilizadores do humor) foi acrescentada a risperidona.

Em estudo aberto, Ghaemi et $\mathrm{al}^{50}$ avaliaram 14 pacientes bipolares I, medicados com risperidona, por um período médio de 6,4 semanas. Ao todo, nove (64\%) pacientes foram considerados respondedores, não mais preenchendo critérios de diagnóstico do DSM-III-R para episódio afetivo. É importante destacar que apenas três pacientes (21\%) não receberam estabilizadores do humor simultaneamente.

Ghaemi et $\mathrm{al}^{51}$ seguiram 12 pacientes bipolares que apresentaram episódios de recidiva mesmo em regime apropriado com estabilizadores do humor, por um período médio de seis meses. Ao regime já em curso, foi acrescentada risperidona em uma dose média de $2,75 \mathrm{mg} /$ dia. Registrou-se uma melhora em quatro $(33 \%)$ pacientes, sendo que três $(25 \%)$ apresentaram uma acentuada melhora, e um (8\%), apenas uma leve melhora.

Em estudo aberto, Vieta et al ${ }^{52}$ avaliaram o efeito da risperidona em dez bipolares, cicladores rápidos, que não responderam ao tratamento com estabilizadores do humor. Nos seis meses de observação, oito (80\%) pacientes apresentaram melhora significativa. Os autores realçam a cautela com que devem ser vistos os resultados, em função das limitações do desenho do estudo e do uso concomitante de estabilizadores do humor e de outros psicotrópicos.

Em estudo retrospectivo, Frazier et al $^{53}$ estudaram um grupo de 28 crianças e adolescentes bipolares, tratados com risperidona por um período médio de seis meses. Apresentaram uma melhora significativa $82 \%$ dos pacientes, sendo que 27 (96\%) utilizaram outras medicações durante o estudo.

Segal et al, ${ }^{54}$ em estudo duplo-cego, avaliaram a eficácia da risperidona $(n=15)$, comparando-a à do haloperidol $(n=15)$ e do lítio $(n=15)$ em pacientes maníacos, por um período de 28 dias. Ao final do estudo, os autores concluíram que as três substâncias apresentaram eficácia semelhante, com melhora sintomática altamente significativa $(\mathrm{p}<0,001)$.

De um modo geral, os estudos com a risperidona se mostraram controversos e com marcantes limitações metodológicas, como pequenas amostras, freqüente uso de medicações concomitantes e alguns estudos retrospectivos, o que dificulta a formulação de qualquer conclusão. Futuros estudos duplocegos, randomizados e que utilizem a monoterapia como estratégia poderão trazer mais esclarecimentos.

\section{Olanzapina}

\section{Fase aguda: mania}

Ao todo, a eficácia da olanzapina foi avaliada em mais de 600 pacientes bipolares na fase maníaca. Na Tabela 1, encontram-se resumidos os principais estudos publicados sobre o uso da olanzapina na mania aguda.

Em 1997, Ravindran et al ${ }^{55}$ relataram o tratamento bem-sucedido com olanzapina de dois pacientes bipolares, dando início a uma série de estudos que culminou com os estudos duplos-cegos controlados e com a aprovação do FDA.

Ketter et al $^{56}$ publicaram o relato de dois pacientes com diagnóstico de transtorno bipolar I, em fase maníaca mista sem sintomas psicóticos, que melhoraram rapidamente quando $10 \mathrm{mg} /$ dia de olanzapina foram adicionados a estabilizadores do humor já em curso. No mesmo ano, McElroy et $\mathrm{al}^{57}$ publicaram o primeiro estudo aberto avaliando pacientes resistentes ao tratamento com medicamentos convencionais, sendo 12 maníacos e dois, depressivos. Dos que estavam em fase maníaca, oito (67\%) apre- 
Tabela 1 - Estudos com a olanzapina no tratamento da mania aguda.

\begin{tabular}{|c|c|c|c|c|c|}
\hline Autor/Ano & Tipo de Estudo & $\mathrm{N}$ & Critério de Resposta & $\%$ Melhora & Monoterapia ou Combinação \\
\hline Ravindran et al., 1997 & Relato de caso & 2 & & $100 \%$ & Monoterapia \\
\hline Ketter et al., 1998 & Relato de caso & 2 & & $100 \%$ & Combinação \\
\hline McElroy et al., 1998 & Estudo aberto & 14 & CGI & $57 \%$ & Combinação \\
\hline Soutullo et al., 1999 & Estudo aberto & 7 & CGI & $71 \%$ & Combinação \\
\hline Tohen et al., 1999 & Duplo cego, placebo controlado & 139 & Y-MRS, PANSS, CGI & $48,6 \%$ & Monoterapia \\
\hline Chang e Ketter, 2000 & Relato de caso & 3 & & $100 \%$ & Combinação \\
\hline Ohaeri, 2000 & Estudo aberto & 8 & PANSS, CGI & $100 \%$ & Monoterapia \\
\hline Tohen et al., 2000 & Duplo cego, placebo controlado & 344 & Y-MRS, HAM-D & $67,7 \%$ & Combinação \\
\hline Tohen et al., 2000 & Duplo cego, placebo controlado & 115 & Y-MRS & $65 \%$ & Monoterapia \\
\hline Total & - & 632 & . & $63,5 \%$ * & \\
\hline
\end{tabular}

sentaram melhora após um mês de tratamento.

Examinando um grupo de 139 pacientes em um estudo duplo-cego, Tohen et $\mathrm{al}^{58}$ os dividiram aleatoriamente em um grupo que fez uso de olanzapina ( $5 \mathrm{mg} / \mathrm{d}$ a $20 \mathrm{mg} / \mathrm{d}$; $\mathrm{n}=70$ ) e outro que recebeu placebo $(n=69)$. A melhora clínica foi definida como uma redução de $50 \%$ ou mais do escore total do Young Mania Rating Scale (YMRS), medido quando da entrada no estudo, momento em que se exigia um escore total de, no mínimo, 20 pontos. Destacam-se algumas características importantes desse estudo, tais como o primeiro estudo duplo-cego placebo controlado da olanzapina em pacientes bipolares, uma amostra com número bastante significativo, avaliação da olanzapina como monoterapia e claros critérios de inclusão e exclusão. O grupo que tomou a olanzapina apresentou uma significante maior taxa de respondedores (48,6\% versus $24,2 \%$; $\mathrm{p}=0,004)$, menos abandonos de tratamento por conta da ineficácia da substância $(28,6 \%$ versus $47,8 \%$; $\mathrm{p}=0,02)$ e maior ganho de peso $(1,65 \mathrm{~kg}$ e $0,44 \mathrm{~kg}$, respectivamente; $\mathrm{p}<0,001)$. Esses autores registraram também uma mesma taxa de respondedores entre os pacientes com ou sem sintomas psicóticos, não aparecendo, portanto, apenas a ação antipsicótica da olanzapina, já amplamente estabelecida, mas também sugerindo um efeito antimaníaco próprio.

Berk et al, ${ }^{59}$ em estudo duplo-cego randomizado, utilizando uma amostra de 30 pacientes bipolares em fase maníaca, concluíram, ao final de quatro semanas, que a olanzapina é provavelmente tão eficaz quanto o lítio no tratamento da mania. Embora não se tenham registrado diferenças entre os dois grupos quanto aos escores finais na BPRS ou no YMRS, o grupo da olanzapina apresentou escores significativamente melhores, naquele período, na escala de gravidade do $\mathrm{CGI}(\mathrm{p}=0,025)$.

Tohen et $\mathrm{al}^{60}$ avaliaram 344 pacientes com transtorno bipolar I em mania aguda ou episódio misto, em estudo duplo-cego randomizado e placebo controlado, utilizando olanzapina (5 $\mathrm{mg} / \mathrm{d}$ a $20 \mathrm{mg} / \mathrm{d}$ ) ou placebo, concomitantemente ao uso de valproato ou lítio, durante seis semanas. Os pacientes em uso de olanzapina apresentaram melhora significantemente superior aos do grupo-placebo, de acordo com a avaliação sintomatológica pelo YMRS (redução do escore $>50 \% ; 67,7 \%$ vs. $44,7 \% ; p=0,003)$. É importante destacar que os pacientes com sintomas depressivos também apresentaram uma melhora superior ao placebo quando avaliados com o Hamilton Depression Rating Scale (HDRS) (43,1\% vs. 9,5\%; $\mathrm{p}=0,006)$. Os efeitos colaterais significativamente mais freqüentes com o uso da olanzapina foram: sonolência, xerostomia, ganho de peso, aumento do apetite e tremores.

O mesmo grupo de pesquisadores,${ }^{61}$ em um outro estudo duplo-cego placebo controlado, avaliou a eficácia da olanzapina ( $5 \mathrm{mg} / \mathrm{d}$ a $20 \mathrm{mg} / \mathrm{d}$ ) em 115 pacientes com episódios maníacos ou mistos, utilizando como critério de melhora clínica uma redução de, pelo menos, 50\% do escore inicial do YMRS. O estudo durou quatro semanas, e os pacientes do grupo da olanzapina apresentaram uma melhora clínica significativamente superior ao placebo já ao final da primeira semana de tratamento $(\mathrm{p}=0,03)$ e se mantiveram assim durante as quatro semanas de observação. Ao final do estudo, 64,8\% apresentaram melhora clínica com a olanzapina, comparado a $42,9 \%$ com o placebo $(\mathrm{p}=0,02)$. Também, um número significativamente superior de pacientes eutímicos foi registrado após o tratamento, no grupo da olanzapina $(61,1 \%$ vs. $35,7 \%$; $p=0,01)$. Mais uma vez, foi registrada uma melhora dos sintomas depressivos moderados ou graves (escore da HDRS $>20$, quando do início do estudo) significativamente maior com a olanzapina $(p=0,05)$. Não foi observada diferença em relação à freqüência de efeitos extrapiramidais, nem quanto ao uso de anticolinérgicos entre os dois grupos $(\mathrm{p}=0,23)$. No grupo que recebeu a olanzapina, foi observado um significativo ganho de peso ao final do estudo $(\mathrm{p}=0,002)$. Comparado ao estudo duplo-cego anteriormente publicado pelo mesmo grupo, o atual apresentou uma duração maior (quatro vs. três semanas), dose inicial de olanzapina maior $(15 \mathrm{mg} / \mathrm{d} v s .10 \mathrm{mg} / \mathrm{d})$, além de ser permitida uma menor dose de benzodiazepínico (aproximadamente metade da dose do estudo prévio). É provável que uma dose inicial maior, ao lado de um maior tempo de tratamento, explique o maior número de respondedores registrados no presente estudo em relação ao anterior $(64,8 \%$ vs. 48,6\%). Entretanto, o maior período de tratamento, assim como uma elevada porcentagem de pacientes que responderam satisfatoriamente a outras medicações em episódios anteriores, pode ter contribuído para uma taxa de resposta mais elevada ao grupo do placebo neste estudo (42,9\% vs. 24,2\%).

Em suma, os estudos relatados anteriormente se mostraram unânimes em apontar a olanzapina como uma medicação segura e eficaz no tratamento da mania aguda. Sugerem também que a olanzapina, quando iniciada em doses mais elevadas, apresenta um início de ação bastante rápido.

\section{Episódio depressivo}

Os antipsicóticos clássicos até então não tiveram lugar entre 
as estratégias para o tratamento das depressões, exceto em associação com antidepressivos, para o tratamento de depressão grave com sintomas psicóticos. Pelo contrário, têm sido descritas como substâncias capazes de piorar, ou mesmo induzir, quadros depressivos. ${ }^{62}$

Embora com evidências muito aquém daquelas apresentadas para o tratamento da mania aguda, a olanzapina tem mostrado alguns resultados promissores, pelo menos como potencializador, para o tratamento de sintomas depressivos. Em 1997, Tollefson et al ${ }^{63}$ avaliaram 1.996 pacientes com diagnóstico de esquizofrenia, transtorno esquizoafetivo ou transtorno esquizofreniforme, que foram medicados com olanzapina $(n=1336)$ ou haloperidol $(n=660)$. A maioria dos pacientes apresentava diagnóstico de esquizofrenia paranóide, com $86 \%$ apresentando curso crônico. Os autores registraram uma inesperada superioridade da olanzapina em relação à avaliação de sintomas depressivos a partir do Montgomery-Asberg Depression Rating Scale (MADRS). Os efeitos relatados chamam a atenção em função de sua magnitude, que, de fato, os aproxima daqueles vistos em ensaios clínicos duplo-cegos com antidepressivos. Utilizando critérios mais restritivos de melhora clínica (redução de 50\% ou mais no escore total do MADRS), mais uma vez, torna-se nítido o possível efeito antidepressivo da olanzapina, com a qual significativamente mais pacientes melhoraram, quando se compara ao uso do haloperidol $(46 \%$ vs. $35 \%$; $\mathrm{p}=0,001)$. Seguem os autores comentando que o perfil farmacodinâmico da olanzapina pode ser o responsável pelo efeito antidepressivo. Visto que há relatos de que a densidade de receptores 5-HT2A no córtex cerebral encontra-se aumentada nos pacientes com transtorno depressivo, a olanzapina, como um potente antagonista em receptores 5-HT2A, pode ter atuado nesses locais de forma similar ao antidepressivo nefazodona, já aprovado pelo FDA para essa indicação.

Weisler et al $^{64}$ relataram uma acentuada melhora em duas pacientes com diagnóstico de depressão grave refratária (uma unipolar, outra bipolar) após a introdução de $10 \mathrm{mg} / \mathrm{d}$ de olanzapina.

Tollefson et al, ${ }^{65}$ em estudo duplo-cego randomizado placebo controlado, avaliaram 335 pacientes com esquizofrenia de curso crônico, com exacerbação aguda, comparando o efeito de haloperidol (10 mg/d a $20 \mathrm{mg} / \mathrm{d})$, olanzapina $(5 \mathrm{mg} / \mathrm{d}, 10 \mathrm{mg} / \mathrm{d}$ ou $15 \mathrm{mg} / \mathrm{d}$ ) e placebo. Houve uma redução estatisticamente significativa no escore do fator ansiedade-depressão do Brief Psychiatric Rating Scale (BPRS) apenas nos subgrupos que utilizaram $10 \mathrm{mg} / \mathrm{d}$ e $15 \mathrm{mg} / \mathrm{d}$ de olanzapina ( $\mathrm{p}=0,042 \mathrm{e} \mathrm{p}=0,046$, respectivamente). Esses achados, embora não oriundos de uma população com diagnótico primário de transtorno depressivo, sugerem que a olanzapina apresenta um efeito antidepressivo próprio, em vez de secundário à redução dos sintomas primários da esquizofrenia, o que certamente ocorreu no grupo do haloperidol que não atingiu significância estatística $(\mathrm{p}=0,108)$. Os autores, ao interpretar a superioridade do possível efeito antidepressivo da olanzapina, postulam que esse efeito devese ao antagonismo em receptores 5-HT2, o que aumentaria a concentração de serotonina na sinapse ou, como maneira alternativa de explicação, o efeito antidepressivo seria mediado in- diretamente pelo antagonismo serotoninérgico, que possibilitaria uma maior liberação de dopamina e noradrenalina no córtex pré-frontal.

Em estudo preliminar retrospectivo com uma pequena amostra de pacientes, Zarate et $\mathrm{al}^{66}$ observaram uma resposta clínica satisfatória em $100 \%$ dos pacientes com depressão bipolar $(n=2)$ e em $50 \%$ dos pacientes com diagnóstico de transtorno esquizoafetivo do tipo depressivo $(n=4)$, quando do uso isolado de olanzapina.

Em estudo duplo-cego randomizado, comparando o uso de risperidona e olanzapina em 339 pacientes com diagnóstico de esquizofrenia, transtorno esquizoafetivo e transtorno esquizofreniforme, Tollefson et $\mathrm{a}^{67}$ observaram uma redução significativa dos sintomas depressivos ao final de oito semanas apenas no grupo de olanzapina $(n=129, p=0,05)$. Essa redução se correlacionou negativamente ao risco de recaída. Visto que as características clínicas e demográficas dos dois subgrupos não apresentaram qualquer diferença significativa, os autores concluem que a presença de efeitos antidepressivos, ao lado de um menor risco de recaída, no grupo da olanzapina, encontra explicação nos diferentes mecanismos de ação observados entre as duas substâncias.

Malhi et al ${ }^{68}$ relataram uma marcante melhora em dois pacientes com diagnóstico de depressão psicótica, anteriormente responsivos apenas à eletroconvulsoterapia, quando da troca do antipsicótico para olanzapina, em adição à venlafaxina já em uso. A melhora se deu já no curso das três primeiras semanas de tratamento.

Ohaeri et al, ${ }^{69}$ em estudo aberto, observando 64 pacientes, sendo que dez com diagnóstico de depressão grave, relataram uma melhora em todos os pacientes deprimidos com o uso da olanzapina. Na maioria dos casos, esses efeitos já eram detectáveis após duas semanas de tratamento.

Tohen et al, ${ }^{61}$ estudando 115 pacientes com diagnóstico de transtorno bipolar em fase maníaca aguda ou mista, distribuídos aleatoriamente e com metodologia duplo-cega para fazer uso de olanzapina ou placebo, observaram melhora estatisticamente significativa no subgrupo de pacientes que apresentou sintomas depressivos moderados ou graves, quando do início do estudo, após a introdução da olanzapina.

Em resumo, foram avaliados sintomas depressivos em diversos transtornos mentais, porém não se procedeu a nenhum estudo controlado que investigasse especificamente o efeito da olanzapina na depressão, seja unipolar ou bipolar. Ressalvando esse crucial fator limitante, a olanzapina pareceu útil, pelo menos como potencializador, no tratamento de sintomas depressivos. Estudos duplo-cegos controlados e dirigidos especificamente a uma amostra de pacientes com transtornos depressivos poderão tornar mais claros esses achados preliminares.

\section{Fase de manutenção}

Os pacientes que, segundo os critérios operacionais das classificações diagnósticas vigentes, apresentam transtorno bipolar compõem um grupo extremamente heterogêneo, fato que não o faz diferir dos diversos outros transtornos mentais. Desse 
modo, o curso do transtorno bipolar é bastante variável, o que torna premente uma conceituação uniformizada acerca do tratamento de manutenção. Na prática clínica, esse corresponde ao período compreendido entre o final de uma fase aguda (remissão completa) até o início de um outro episódio, seja maníaco ou depressivo.

Dadas as diversas dificuldades metodológicas em avaliar a eficácia de um determinado tratamento de manutenção no transtorno bipolar, a literatura se mostra bastante carente, mesmo quando dirigida às medicações já consagradas quanto ao uso nas fases agudas. Para complicar, em função de a maioria dos estudos terem sido publicados antes de 1975, critérios operacionais de diagnóstico ou entrevistas estruturadas ${ }^{10}$ não eram utilizados.

A despeito do uso generalizado de antipsicóticos no transtorno bipolar, não se encontrou estudo controlado que avaliasse sua eficácia no tratamento de manutenção do transtorno bipolar. Dada essa carência de estudos controlados e endereçados à avaliação de sua eficácia em longo prazo no transtorno bipolar, além de evidências de que os benefícios da olanzapina são melhor notados quando em associação com um estabilizador do humor, há grande interesse em examinar o papel potencial de estratégias que envolvam antipsicóticos atípicos, como a olanzapina, em estudos em longo prazo nos quais se possa examinar uma possível ação primária como estabilizador do humor, seja em monoterapia ou como agente potenciador de outros estabilizadores do humor.

\section{Há preditores de uma boa resposta à olanzapina em paci- entes bipolares?}

Mesmo a resposta à medicação de referência para o tratamento do transtorno bipolar (o lítio) tem se mostrado bastante variável nos diversos estudos clínicos, de modo a apresentar um spectrum que varia de uma excelente resposta, com uma remissão pronta e completa, até uma completa resistência ao tratamento, quando não se registra qualquer mudança na freqüência, na gravidade ou na duração dos episódios. ${ }^{70,71} \mathrm{Na}$ verdade, o lítio parece ter uma melhor eficácia nos pacientes que apresentam um quadro "clássico" de transtorno bipolar: com história familiar positiva, sintomas do tipo grandiosidade, euforia e um rápido início do quadro maníaco, seguido de um quadro depressivo retardado. Os quadros "atípicos" caracterizados por sintomas psicóticos incongruentes, depressões graves, ciclagem rápida ou episódios mistos, relacionam-se a uma resposta pobre..$^{71,72}$ Outros fatores, como curso contínuo, história familiar positiva para esquizofrenia, abuso de álcool ou drogas, marcada predominância de episódios maníacos e gravidade dos episódios também parecem estar associados a um pior prognóstico. ${ }^{73}$

Coryell et al, ${ }^{74}$ conduzindo o maior estudo de follow-up em pacientes bipolares, encontraram como fatores preditores de um prognóstico pior apenas alcoolismo ativo e baixos níveis de funcionamento social nos cinco anos prévios. Ressaltam também que o conhecimento científico acumulado nessa área é escasso, de modo que este foi apenas o segundo estudo com mais de 10 anos de seguimento, limitando as conclusões que possam ser obtidas acerca do tema.
Ao avaliar, retrospectivamente, 145 pacientes que fizeram uso de quetiapina para o tratamento do transtorno bipolar, surpreendentemente, Zarate et $\mathrm{a}^{75}$ encontraram o diagnóstico de depressão com sintomas psicóticos $(\mathrm{p}=0,02)$ e uma mais longa duração do transtorno $(\mathrm{p}=0,03)$ como fatores associados a uma pior resposta à quetiapina.

Em estudo único, Zarate et $\mathrm{al}^{66}$ avaliaram, retrospectivamente, 150 pacientes com transtornos do humor com sintomatologia psicótica, recentemente tratados com olanzapina. Ressaltando as limitações do estudo, os autores relatam interessantes achados acerca dos preditores de uma boa resposta à olanzapina. Ao contrário da hipótese inicial, não houve diferença na avaliação da eficácia entre os pacientes depressivos com ou sem sintomas psicóticos, evidenciando, mais uma vez, uma possível propriedade antidepressiva independentemente da presença de sintomas psicóticos. A olanzapina também apresentou uma maior probabilidade de êxito terapêutico em pacientes mais jovens, de sexo feminino, com menor tempo de doença e com um menor intervalo entre o início do episódio e o uso da olanzapina e que a utilizaram por mais tempo.

Concluindo, se faz necessário investigar, de maneira mais sistemática, possíveis fatores preditores de resposta de pacientes bipolares à olanzapina.

\section{Diretrizes clínicas para o uso da olanzapina no transtorno bipolar}

Tomando por base os diversos estudos revisados no presente trabalho, pode-se traçar algum esboço de diretrizes clínicas, embora, pelo pouco tempo de publicação dos primeiros dados e por sua conseqüente escassez, tais diretrizes não devam ser tomadas como algo estabelecido. Pelo contrário, os dados que as sustentam são pouco robustos e aguardam um maior número de estudos controlados para sua eventual confirmação.

Desse modo, no transtorno bipolar, a olanzapina parece mais eficaz quando usada em doses maiores $(10 \mathrm{mg} / \mathrm{d}$ a 20 $\mathrm{mg} / \mathrm{d}$ ), já iniciando com cerca de $15 \mathrm{mg} / \mathrm{d}$, pois possivelmente garante um início de ação mais rápido, o que conferiria uma potencial vantagem dessa medicação em relação aos estabilizadores do humor já estabelecidos. Por questões metodológicas, o tempo mínimo de uso, nos estudos publicados, foi de três semanas, porém certamente este não é o tempo ideal; em vez disso, parece recomendável que se mantenha o uso por um período, no mínimo, igual ao do próprio episódio. Em função de sua meia-vida, de cerca de 33 horas, pode ser administrada em dose única diária, à noite, de modo a se beneficiar do efeito colateral sedativo. A ausência de registro de interações medicamentosas relevantes com outros psicotrópicos constitui um importante fator, especialmente nos grupos de pacientes com características especiais, como hepatopatas, cardiopatas, nefropatas e idosos.

\section{Conclusão}

A despeito do considerável progresso no tratamento psicofarmacológico do transtorno bipolar, os antipsicóticos ainda constituem importantes estratégias na condição de medicações coadjuvantes para um grande número de pacientes, 
mesmo sabendo do significativo risco associado ao uso dos antipsicóticos clássicos. ${ }^{24}$

Com mais de 600 pacientes avaliados na fase maníaca, a olanzapina se mostrou eficaz, como monoterapia ou em associação, em 63,5\% (média ponderada dos diversos estudos) dos casos, número que não se diferencia dos relatados para o lítio, medicação de referência para o tratamento do transtorno bipolar.

Por outro lado, o lítio administrado isoladamente tem beneficiado uma parcela cada vez menor de pacientes com transtorno bipolar, possivelmente em função da adoção, por parte das classificações atuais, de critérios diagnósticos mais abrangentes e por não haver muita ênfase ao curso do transtorno na formulação do diagnóstico. ${ }^{2}$ Seguramente, um subgrupo de pacientes verdadeiramente refratários ao tratamento de forma isolada com o lítio, supostamente pacientes mais graves ou com sintomatologia mais atípica, necessita da associação de antipsicóticos para alcançar melhores resultados, seja na mania aguda ou, mais raramente, na fase de manutenção. Não se pode deixar de considerar a possibilidade de um início de ação mais precoce com o uso de antipsicóticos na fase maníaca aguda. ${ }^{25}$ Freqüentemente, este é um fator decisivo em relação à prevenção de internações psiquiátricas, com óbvio impacto no bem-estar do paciente e de familiares, assim como no custo do tratamento.

Com um perfil de efeitos colaterais substancialmente mais favorável, os antipsicóticos atípicos se apresentam como um importante avanço na resolução do impasse previamente criado pela utilização de antipsicóticos tradicionais: uma eficácia comprovada dos antipsicóticos, que era acompanhada, invariavelmente, de um elevado risco de efeitos colaterais, incluindo os irreversíveis. Dentro dessa perspectiva, a olanzapina alia um perfil de segurança significativamente melhor a uma eficácia comprovada no tratamento da mania aguda, o que ainda não foi estabelecido em relação a outros antipsicóticos atípicos.
A idéia sobre o possível efeito antidepressivo dos antipsicóticos atípicos apóia-se apenas em dados indiretos e em relatos de caso, logo, com um poder conclusivo restrito. As mesmas limitações estão presentes em sua avaliação como estratégia no tratamento de pacientes refratários, assim como em sua utilidade na fase de manutenção do tratamento de pacientes bipolares. Além disso, ainda não se conhece o perfil do paciente que necessita de um antipsicótico em sua prescrição como estratégia em longo prazo, porém, essa decisão no planejamento terapêutico torna-se menos premente quando da utilização de um antipsicótico atípico, ou seja, as diretrizes de utilização em doses e tempo mínimos, assim como a rígida monitoração acerca de graves efeitos colaterais e o uso "só em última instância" não se aplicam a esse grupo de medicações.

Fazem-se necessárias investigações controladas acerca das possíveis qualidades antidepressivas e de estabilização do humor dos antipsicóticos atípicos, trajetória que não se espera rápida em função de um variado arsenal farmacológico já existente para esses fins. No entanto, considerando-se a enorme relevância clínica do transtorno bipolar e o fato de que uma percentagem grande desses pacientes não responde satisfatoriamente às medicações indicadas para o tratamento do transtorno, tais estudos serão muito importantes e são ansiosamente aguardados.

Em suma, a olanzapina se mostrou eficaz e segura no tratamento da mania aguda, sendo inclusive recentemente aprovada pelo FDA para essa indicação, o que pode se tornar verdadeiro também para outros antipsicóticos atípicos no futuro. Entretanto, fazem-se necessários estudos adicionais acerca do(s) subgrupo(s) de pacientes que mais se beneficia(m) com seu uso, seja isolado ou em associação, por do seu uso no tratamento de pacientes refratários, com seu uso potencial no tratamento de sintomas depressivos e como medicação de manutenção para estabilização do humor.

\section{Referências}

1. Calabrese JR, Woyshville MJ. Lithium therapy: limitations and alternatives in the treatment of bipolar disorders. Ann Clin Psychiatry. 1995;7:103-12.

2. Gershon S, Soares JC. Current therapeutic profile of lithium. Arch Gen Psychiatry. 1997;54:16-20.

3. Peselow ED, Fieve RR, Difiglia C, Sanfilipo MP. Lithium prophylaxis of bipolar illness. The value of combination treatment. Br J Psychiatry. 1994;164:208-14.

4. Goldberg JF, Harrow M, Leon AC. Lithium treatment of bipolar affective disorders under naturalistic followup conditions. Psychopharmacol Bull. 1996;32:47-54.

5. Keck PE, McElroy SL. Outcome in the pharmacologic treatment of bipolar disorder. J Clin Psychopharmacol. 1996;16:15S-23S.

6. Post RM, Uhde TW, Roy-Byrne PP, Joffe RT. Correlates of antimanic response to carbamazepine. Psychiatry Res. 1987;21:71-83.

7. McElroy SL, Keck PE, Pope HG, Hudson JI. Valproate in the treatment of bipolar disorder: literature review and clinical guidelines. $J$ Clin Psychopharmacol. 1992;12:42S-52S.

8. Pope HG, McElroy SL, Keck PE, Hudson JI. Valproate in the treatment of acute mania. A placebo-controlled study. Arch Gen Psychiatry. 1991;48:62-8.

9. Bowden CL, Brugger AM, Swann AC, Calabrese J R, Janicak P G, Petty F, et al. Efficacy of divalproex vs lithium and placebo in the treatment of mania. The Depakote Mania Study Group. Jama. 1994;271:918-24.

10. Bowden CL, Brugger AM, Swann AC, Calabrese J R, Janicak P G, Petty $\mathrm{F}$, et al. Efficacy of divalproex vs lithium and placebo in the treatment of mania. The Depakote Mania Study Group. Jama. 1994;271:918-24.

11. Bansil RK. Clonazepam and bipolar affective disorder. J Clin Psychiatry. 1990;51:522-3.

12. Sachs GS. Use of clonazepam for bipolar affective disorder. J Clin Psychiatry. 1990;51 Suppl:31-4; discussion 50-3.

13. Sachs GS, Rosenbaum JF, Jones L. Adjunctive clonazepam for maintenance treatment of bipolar affective disorder. J Clin Psychopharmacol. 1990;10:42-7.

14. Aronson TA, Shukla S, Hirschowitz J. Clonazepam treatment of five lithium-refractory patients with bipolar disorder. Am J Psychiatry. 1989;146:77-80.

15. Bradwejn J, Shriqui C, Koszycki D, Meterissian G. Double-blind comparison of the effects of clonazepam and lorazepam in acute mania. $J$ Clin Psychopharmacol. 1990;10:403-8.

16. Jobling M, Stein G. Lorazepam in resistant mania. Lancet. 1986;1:510.

17. Prien RF, Caffey EM, Klett CJ. Comparison of lithium carbonate and chlorpromazine in the treatment of mania. Report of the Veterans Administration and National Institute of Mental Health Collaborative Study Group. Arch Gen Psychiatry. 1972;26:146-53. 
18. McElroy SL, Keck PE. Pharmacologic agents for the treatment of acute bipolar mania. Biol Psychiatry. 2000;48:539-57.

19. Hopkins HS, Gelenberg AJ. Treatment of bipolar disorder: how far have we come? Psychopharmacol Bull. 1994;30:27-38.

20. Keck PE, McElroy SL, Strakowski SM, Balistreri TM, Kizer DI, West SA. Factors associated with maintenance antipsychotic treatment of patients with bipolar disorder. J Clin Psychiatry. 1996;57:147-51.

21. Remington G, Kapur S. Atypical antipsychotics: are some more atypical than others? Psychopharmacology (Berl). 2000;148:3-15.

22. Dawkins K, Lieberman JA, Lebowitz BD, Hsiao JK. Antipsychotics: Past and Future: National Institute of Mental Health Division of Services and Intervention Research Workshop, July 14, 1998. Schizophr Bull. 1999;25:395-405

23. Shelton RC, Tollefson GD, Tohen M, Stahl S, Gannon K S, Jacobs T G, et al. A novel augmentation strategy for treating resistant major depression. Am J Psychiatry. 2001;158:131-4.

24. Soares JC, Mallinger AG, Gershon S. The role of antipsychotic agents in the treatment of bipolar disorder patients. Int Clin Psychopharmacol. 1997;12:65-76.

25. Sernyak MJ, Griffin RA, Johnson RM, Pearsall HR, Wexler BE, Woods SW. Neuroleptic exposure following inpatient treatment of acute mania with lithium and neuroleptic. Am J Psychiatry. 1994;151:133-5.

26. Mukherjee S, Rosen AM, Caracci G, Shukla S. Persistent tardive dyskinesia in bipolar patients. Arch Gen Psychiatry. 1986;43:342-6.

27. Kane JM. The role of neuroleptics in manic-depressive illness. J Clin Psychiatry. 1988;49 Suppl:12-4.

28. Dinan TG, Kohen D. Tardive dyskinesia in bipolar affective disorder: relationship to lithium therapy. Br J Psychiatry. 1989;155:55-7.

29. Esparon J, Kolloori J, Naylor GJ, McHarg AM, Smith AH, Hopwood SE. Comparison of the prophylactic action of flupenthixol with placebo in lithium treated manic-depressive patients. $\mathrm{Br} J$ Psychiatry. 1986;148:723-5.

30. Soares JC, Barwell M, Mallinger AG, Kupfer DJ, Frank E. Adjunctive antipsychotic use in bipolar patients: an open 6-month prospective study following an acute episode. J Affect Disord. 1999;56:1-8.

31. Hippius H. The history of clozapine. Psychopharmacology (Berl). 1989;99:S3-5.

32. Meltzer HY. Clinical studies on the mechanism of action of clozapine: the dopamine-serotonin hypothesis of schizophrenia. Psychopharmacology (Berl). 1989;99:S18-27.

33. Muller P, Heipertz R. [Treatment of manic psychosis with clozapine (author's transl)]. Fortschr Neurol Psychiatr Grenzgeb. 1977;45:420-4.

34. Calabrese JR, Meltzer HY, Markovitz PJ. Clozapine prophylaxis in rapid cycling bipolar disorder. J Clin Psychopharmacol. 1991;11:396-7.

35. Frankenburg FR. Clozapine and bipolar disorder. J Clin Psychopharmacol. 1993;13:289-290.

36. Suppes T, Phillips KA, Judd CR. Clozapine treatment of nonpsychotic rapid cycling bipolar disorder: a report of three cases. Biol Psychiatry. 1994;36:338-40.

37. Frye MA, Altshuler LL, Bitran JA. Clozapine in rapid cycling bipolar disorder. J Clin Psychopharmacol. 1996;16:87-90.

38. Mahmood T, Devlin M, Silverstone T. Clozapine in the management of bipolar and schizoaffective manic episodes resistant to standard treatment. Aust N Z J Psychiatry. 1997;31:424-6.

39. Calabrese JR, Kimmel SE, Woyshiville MJ. Clozapine in treatment refractory mania. Annual meeting of the American College of Neuropsychopharmacology. Honolulu, Hawaii. Dec 14, 1993.

40. Banov MD, Zarate CA, Jr., Tohen M, Scialabba D, Wines JD, Jr., Kolbrener M, et al. Clozapine therapy in refractory affective disorders: polarity predicts response in long-term follow-up. J Clin Psychiatry. 1994;55:295-300.

41. Zarate CA, Jr., Tohen M, Banov MD, Weiss MK, Cole JO. Is clozapine a mood stabilizer? J Clin Psychiatry. 1995;56:108-12.

42. Calabrese JR, Kimmel SE, Woyshville MJ, Rapport DJ, Faust CJ, Thompson PA, et al. Clozapine for treatment-refractory mania. Am J Psychiatry. 1996;153:759-64

43. Ciapparelli A, Dell'Osso L, Pini S, Chiavacci MC, Fenzi M, Cassano GB. Clozapine for treatment-refractory schizophrenia, schizoaffective disorder, and psychotic bipolar disorder: a 24-month naturalistic study. J Clin Psychiatry. 2000;61:329-34.
44. Guille C, Sachs GS, Ghaemi SN. A naturalistic comparison of clozapine, risperidone, and olanzapine in the treatment of bipolar disorder. $J$ Clin Psychiatry. 2000;61:638-42.

45. Keck PE, Jr., Wilson DR, Strakowski SM, McElroy SL, Kizer DL, Balistreri $\mathrm{TM}$, et al. Clinical predictors of acute risperidone response in schizophrenia, schizoaffective disorder, and psychotic mood disorders. J Clin Psychiatry. 1995;56:466-70.

46. Schaffer CB, Schaffer LC. The use of risperidone in the treatment of bipolar disorder. J Clin Psychiatry. 1996;57:136.

47. Diaz SF. Mania associated with risperidone use. J Clin Psychiatry. 1996;57:41-2.

48. Barkin JS, Pais VM, Gaffney MF. Induction of mania by risperidone resistant to mood stabilizers. J Clin Psychopharmacol. 1997;17:57-8.

49. McIntyre R, Young LT, Hasey G, Patelis-Siotis I, Jones BD. Risperidone treatment of bipolar disorder. Can J Psychiatry. 1997;42:88-90.

50. Ghaemi SN, Sachs GS, Baldassano CF, Truman CJ. Acute treatment of bipolar disorder with adjunctive risperidone in outpatients. Can $J$ Psychiatry. 1997;42:196-9.

51. Ghaemi SN, Sachs GS. Long-term risperidone treatment in bipolar disorder: 6-month follow up. Int Clin Psychopharmacol. 1997;12:333-8.

52. Vieta E, Gasto C, Colom F, Martinez A, Otero A, Vallejo J. Treatment of refractory rapid cycling bipolar disorder with risperidone. $J$ Clin Psychopharmacol. 1998;18:172-4.

53. Frazier JA, Meyer MC, Biederman J, Wozniak J, Wilens TE, Spencer TJ, et al. Risperidone treatment for juvenile bipolar disorder: a retrospective chart review. J Am Acad Child Adolesc Psychiatry. 1999;38:960-5.

54. Segal J, Berk M, Brook S. Risperidone compared with both lithium and haloperidol in mania: a double-blind randomized controlled trial. Clin Neuropharmacol. 1998;21:176-80.

55. Ravindran AV, Jones BW, al-Zaid K, Lapierre YD. Effective treatment of mania with olanzapine: 2 case reports. J Psychiatry Neurosci. 1997;22:345-6.

56. Ketter TA, Winsberg ME, DeGolia SG, Dunai M, Tate DL, Strong CM. Rapid efficacy of olanzapine augmentation in nonpsychotic bipolar mixed states. J Clin Psychiatry. 1998;59:83-5.

57. McElroy SL, Frye M, Denicoff K, Altshuler L, Nolen W, Kupka R, et al Olanzapine in treatment-resistant bipolar disorder. J Affect Disord. 1998;49:119-22.

58. Tohen M, Sanger TM, McElroy SL, et Tollefson GD, Chengappa KN, Daniel DG, et al. Olanzapine versus placebo in the treatment of acute mania. Olanzapine HGEH Study Group. Am J Psychiatry. 1999;156:702-9.

59. Berk M, Ichim L, Brook S. Olanzapine compared to lithium in mania: a double-blind randomized controlled trial. Int Clin Psychopharmacol. 1999;14:339-43.

60. Tohen M, Jacobs TG, Meyers TM. Efficacy of olanzapine combined with mood stabilizers in the treatment of bipolar disorder. 2000 APA Annual Meeting. Chicago, IL. 2000.

61. Tohen M, Jacobs TG, Grundy SL, McElroy SL, Banov MC, Janicak PG, et al. Efficacy of olanzapine in acute bipolar mania: a double-blind, placebocontrolled study. The Olanzipine HGGW Study Group. Arch Gen Psychiatry. 2000;57:841-9.

62. Harrow M, Yonan CA, Sands JR, Marengo J. Depression in schizophrenia: are neuroleptics, akinesia, or anhedonia involved? Schizophr Bull. 1994;20:327-38.

63. Tollefson GD, Beasley CM, Tran PV, Greaney JJ, Berman A, Pike S, et al. Olanzapine versus haloperidol in the treatment of schizophrenia and schizoaffective and schizophreniform disorders: results of an international collaborative trial. Am J Psychiatry. 1997;154:457-65.

64. Weisler RH, Ahearn EP, Davidson JR, Wallace CD. Adjunctive use of olanzapine in mood disorders: five case reports. Ann Clin Psychiatry. 1997:9:259-62.

65. Tollefson GD, Sanger TM, Beasley CM, Tran PV. A double-blind, controlled comparison of the novel antipsychotic olanzapine versus haloperidol or placebo on anxious and depressive symptoms accompanying schizophrenia. Biol Psychiatry. 1998;43:803-10.

66. Zarate CA, Narendran R, Tohen M, Greaney JJ, Berman A, Pike S, et al Clinical predictors of acute response with olanzapine in psychotic mood disorders. J Clin Psychiatry. 1998;59:24-8.

67. Tollefson GD, Andersen SW, Tran PV. The course of depressive symptoms in predicting relapse in schizophrenia: a double-blind, randomized comparison of olanzapine and risperidone. Biol Psychiatry. 1999;46:365-73. 
68. Malhi GS, Checkley SA. Olanzapine in the treatment of psychotic depression. Br J Psychiatry. 1999;174:460.

69. Ohaeri JU. Naturalistic study of olanzapine in treatment-resistant schizophrenia and acute mania, depression and obsessional disorder. East Afr Med J. 2000;77:86-92.

70. Abou-Saleh MT, Coppen A. Who responds to prophylactic lithium? J Affect Disord. 1986;10:115-25.

71. Soares JC, Gershon S. The lithium ion: a foundation for psychopharmacological specificity. Neuropsychopharmacology. 1998;19:167-82.

72. Maj M. Clinical prediction of response to lithium prophylaxis in bipolar patients: the importance of the previous pattern of course of the illness. Clin Neuropharmacol. 1990;13:S66-70.

73. Yazici O, Kora K, Ucok A, Tunali D, Turan N. Predictors of lithium prophylaxis in bipolar patients. J Affect Disord. 1999;55:133-42.

74. Coryell W, Turvey C, Endicott J, Leon AC, Mueller T, Solomon D, et al. Bipolar I affective disorder: predictors of outcome after 15 years. $J$ Affect Disord. 1998;50:109-16.
75. Zarate CA, Rothschild A, Fletcher KE, Madrid A, Zapatel J. Clinical predictors of acute response with quetiapine in psychotic mood disorders. J Clin Psychiatry. 2000;61:185-9.

\section{Correspondência}

Acioly L T Lacerda

Neurochemical Brain Imaging Laboratory

Western Psychiatric Institute and Clinic

University of Pittsburgh School of Medicine

3811 O'Hara St, Pittsburgh, PA 15213

Tel.: (00xx1) (412) 624-4452

E-mail: lacerdaal@msx.upmc.edu 